\title{
The Liprin Homology Domain Is Essential for the Homomeric Interaction of SYD-2/Liprin- $\alpha$ Protein in Presynaptic Assembly
}

\author{
Hidenori Taru ${ }^{1,2,3}$ and Yishi Jin ${ }^{3,4,5}$ \\ ${ }^{1}$ Creative Research Institution and 2Laboratory of Neuronal Cell Biology, Graduate School of Pharmaceutical Sciences, Hokkaido University, Sapporo 001- \\ 0021, Japan, and ${ }^{3}$ Section of Neurobiology, Division of Biological Sciences, ${ }^{4}$ Howard Hughes Medical Institute, and ${ }^{5}$ Department of Cellular and Molecular \\ Medicine, School of Medicine, University of California, San Diego, California 92093
}

\begin{abstract}
Synapses are asymmetric structures that are specialized for neuronal signal transduction. A unique set of proteins is present at the presynaptic active zone, which is a core structure essential for neurotransmitter release. In Caenorhabditis elegans HSN neurons, SYD-2, a Liprin- $\alpha$ family protein, acts together with a GAP protein SYD-1 to promote presynaptic assembly. Previous studies have shown that elevating the activity of $s y d-2$ can bypass the requirement of $s y d-1$. Liprin- $\alpha$ proteins are composed of coiled-coil-rich regions in the $\mathrm{N}$-terminal half, which mediate interactions with adapter proteins at the presynaptic active zone, and three SAM domains in the C terminus, which bind proteins such as LAR receptor tyrosine phosphatase. To address the molecular mechanism by which SYD-2 activity is regulated, we performed structure-function studies. By monitoring the ability of SYD-2 transgenes to rescue syd-2(lf) and to suppress syd-1 (lf) phenotypes in HSN neuron synapses, we identified the N-terminal half of SYD-2 as minimally required for rescuing syd-2(lf) phenotypes. A highly conserved short coiled-coil segment named Liprin Homology 1 (LH1) domain is both necessary and sufficient to suppress syd-1(lf) defects. We show that the LH1 domain forms a dimer and promotes further oligomerization and/or complex formation of SYD-2/Liprin- $\alpha$ proteins. The role of the LH1 domain in presynaptic assembly can be partially complemented by artificial dimerization. These findings suggest a model by which the self-assembly of SYD-2/Liprin- $\alpha$ proteins mediated by the coiled-coil LH1 domain is one of the key steps to the accumulation of presynaptic components at nascent synaptic junctions.
\end{abstract}

\section{Introduction}

Chemical synapses consist of a presynaptic terminal, a synaptic cleft, and a postsynaptic specialization. The presynaptic active zone, through which neurotransmitters are released, accumulates a distinct set of proteins (Zhai and Bellen, 2004). During presynaptic differentiation, these proteins are delivered by vesicle-based transport and assembled at nascent synaptic sites to form an organized nanostructure (Jin and Garner, 2008). The

Received Jan. 1, 2011; revised Aug. 30, 2011; accepted Sept. 5, 2011.

Author contributions: H.T. and Y.J. designed research; H.T. performed research; H.T. analyzed data; H.T. and Y.J. wrote the paper.

This work was supported by grants-in-aid for Young Scientists from the Ministry of Education, Culture, Sports, Science, and Technology (21790057, 23790070), The Akiyama Life Science Foundation, Suzuken Memorial Foundation, Postdoctoral Fellowships from Japanese Society for Promoting Science, a start-up fund of the Leader Development System in the Basic Interdisciplinary Research Areas at Hokkaido University to H.T., and NIH Grant NS035546 to Y.J.Y.J. is an investigator of the Howard Hughes Medical Institute. We thank K. Shen (Stanford University) for kyls 235 and wyls 22 markers, Punc- 86 plasmid, and communicating unpublished data, the C. elegans gene knockout consortium and C. elegans genetic center for syd-2(ok217), R. Jin (Sanford-Burnham Institute), S. Adams (University of California, San Diego), and T. Suzuki (Hokkaido University) for equipment, materials, and technical advice for biochemical analyses, and Y. Dai and members of the Jin and Chisholm laboratories and the Taru Lab for technical advice and support.

The authors declare no competing financial interests.

Correspondence should be addressed to Hidenori Taru, Creative Research Institution, Hokkaido University, Kita21-Nishi10, Kita-ku, Sapporo 001-0021, Japan. E-mail: taru@cris.hokudai.ac.jp.

DOI:10.1523/JNEUROSCI.0002-11.2011

Copyright $\odot 2011$ the authors $\quad 0270-6474 / 11 / 3116261-08 \$ 15.00 / 0$ molecular mechanisms of how the assembly of presynaptic components is initiated and regulated remain unknown.

Liprin- $\alpha$ family proteins are scaffolding proteins (Spangler and Hoogenraad, 2007). The N-terminal halves of the proteins are coiled-coil rich, and contain two highly conserved segments called "Liprin- $\alpha$ Homology regions 1 (LH1) and 2 (LH2)" (Schoch et al., 2002). The C-terminal halves contain three conserved Sterile- $\alpha$-motif (SAM) domains, often referred as to LHD (for Liprin Homology Domain), which bind LAR-type receptor protein tyrosine phosphatase (Serra-Pagès et al., 1998). The $\mathrm{N}$-terminal of Liprin- $\alpha$ can bind multiple proteins, including RIM (Schoch et al., 2002) and ELKS/ERC/CAST (Ko et al., 2003), which play distinct roles in presynaptic vesicle release. The in vivo functions of Liprin- $\alpha$ in synapses have been best studied in invertebrates. Caenorhabditis elegans SYD-2 and Drosophila Liprin- $\alpha$ are localized at the center of presynaptic active zones and required for the active zone organization in various types of neurons (Zhen and Jin, 1999; Kaufmann et al., 2002; Yeh et al., 2005; Fouquet et al., 2009; Stigloher et al., 2011).

In C. elegans hermaphrodite-specific motor neurons (HSNs), SYD-2/Liprin- $\alpha$ and a RhoGAP protein SYD-1 are essential for the assembly of downstream presynaptic components. The activity of SYD-2 depends in part on ELKS-1 and a novel negative regulator RSY-1 (Dai et al., 2006; Patel et al., 2006; Patel and Shen, 2009). We have previously reported that a missense mutation, syd-2(ju487gf), alters Arg184 to Cys in the LH1 domain, and 

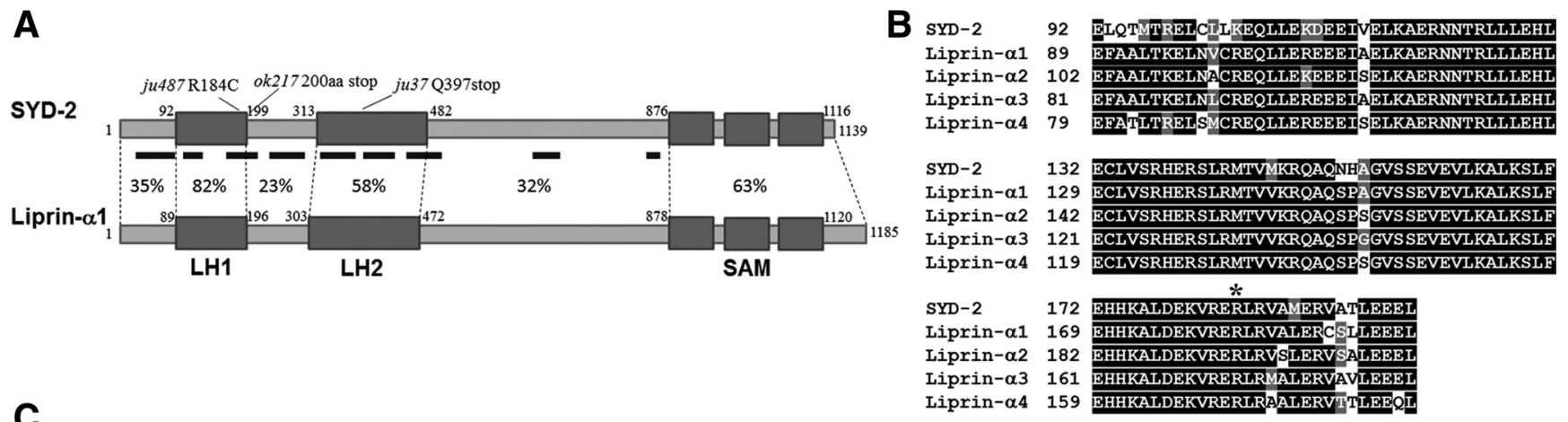

C

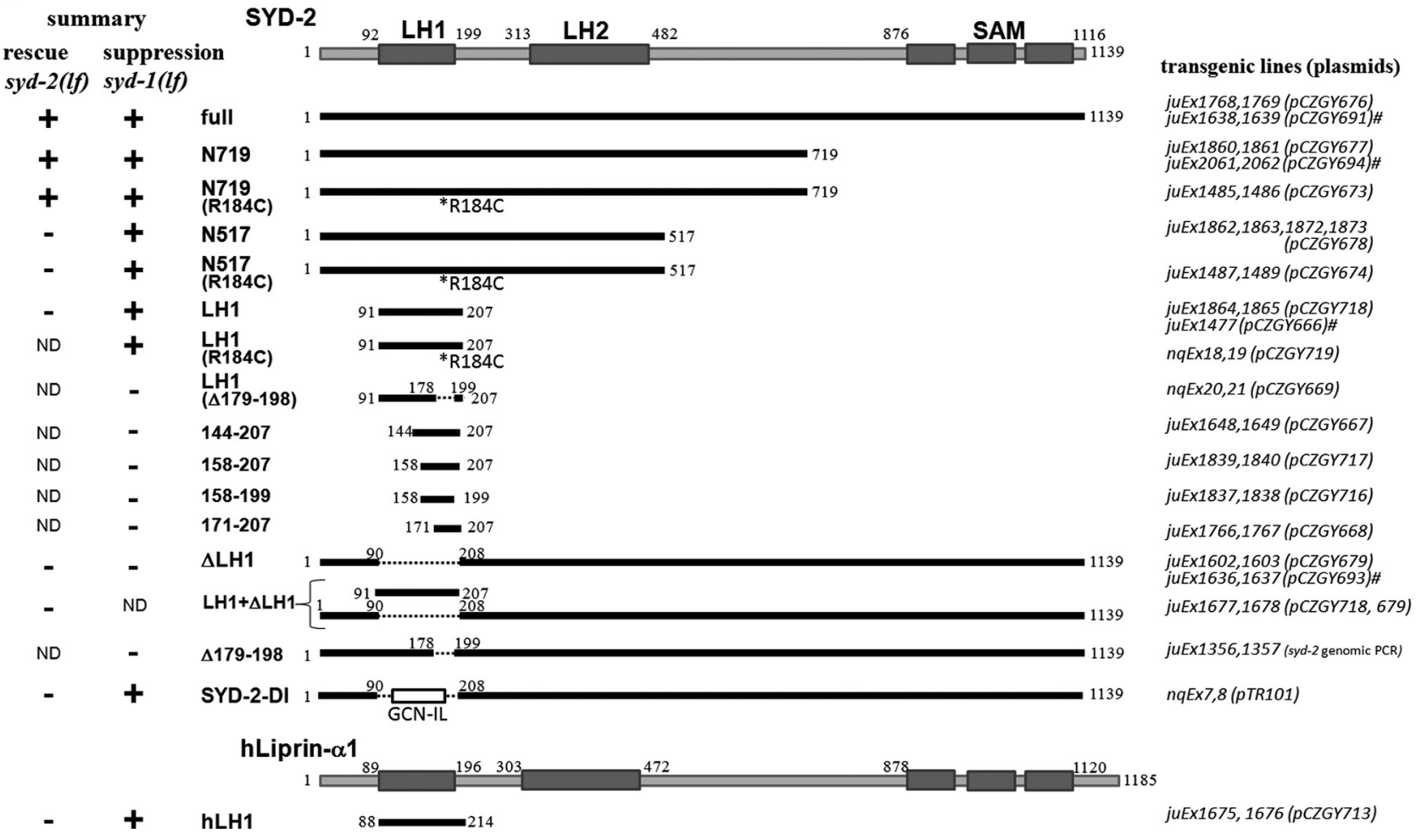

Figure 1. SYD-2/Liprin- $\alpha$ proteins and summary of transgenic analyses. $A$, Illustration of the domain structure of C. elegans SYD- 2 and human Liprin- $\alpha 1$ proteins. Homology (percentage of identical residues) and predicted coiled-coil segments (underline) are indicated. $\boldsymbol{B}$, Sequence alignment of the LH1 domains of SYD- 2 and human Liprin- $\alpha$ proteins. The residues with black and gray background represent identical and similar residues, respectively. Asterisk indicates Arg184 residue of SYD-2. C, Summary of transgenic structural-functional analyses of SYD-2. The structure of SYD-2/Liprin- $\alpha$ constructs and the presence ( + ) or absence $(-)$ of the ability to rescue syd-2(If) or suppress syd-1(If) phenotypes are indicated. Transgenic lines and plasmids used for pan-neural and HSN expression (\#) are listed.

causes a gain-of-function effect such that the mutant protein can promote assembly of synaptic components even in the absence of SYD-1 (Dai et al., 2006). Overexpression of SYD-2 can also bypass the requirement of SYD-1 (Dai et al., 2006; Patel et al., 2006). These observations show that the activity of SYD-2 is critical for proper presynaptic assembly. Homomeric interactions of SYD-2/Liprin- $\alpha$ have been previously reported (Serra-Pagès et al., 1998; Wagner et al., 2009; Astigarraga et al., 2010). However, it remains poorly understood whether the homomeric interaction is important for their function and how it is mediated.

Here, we performed transgenic and biochemical studies to address the role of the conserved LH1 domain of SYD-2. Our data supports a proposal that self-assemble property of SYD-2/ Liprin- $\alpha$ mediated by the dimerization of LH1 domain promotes a higher order of oligomerization and clustering, which is essential for presynaptic formation.

\section{Materials and Methods}

Plasmid construction. The expression plasmids were generated following standard methods or using Gateway cloning system (Invitrogen). Prgef-1 and $P u n c-86$ promoters were used for pan-neuronal and HSN expressions, respectively. Mutant SYD-2 or human Liprin- $\alpha 1$ A constructs were made by PCR using cDNAs as templates (Serra-Pagès et al., 1998; Zhen and Jin, 1999), and verified by sequencing. Oligo nucleotides for GCN-IL peptide (RMKQLEDKIEELLSKIYHLENEIARLKKLIGER) with spacers (GGSGG) were used to generate SYD-2-DI. GIT-1 cDNA (Source BioScience) was cloned into pPD49.26 with YFP as C-terminal tag driven by Punc-86 promoter. Detailed information for plasmids is available upon request.

C. elegans genetics and transgenic analysis. C. elegans strains were maintained on NGM plates at $20-22.5^{\circ} \mathrm{C}$ as described previously (Brenner, 1974). The mutations and marker transgenes used were as follows: syd1(ju2)II (Hallam et al., 2002), syd-2(ju37)X (Zhen and Jin, 1999), syd-

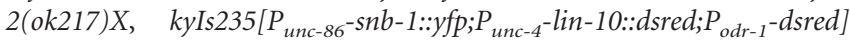

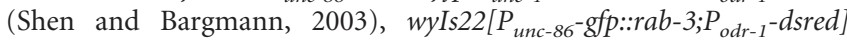


A

$B$ background

wt

syd-2(ju37)

SYD-2

\begin{tabular}{lllll}
\hline- & full & N719 & N517 & $\Delta L H 1$
\end{tabular}
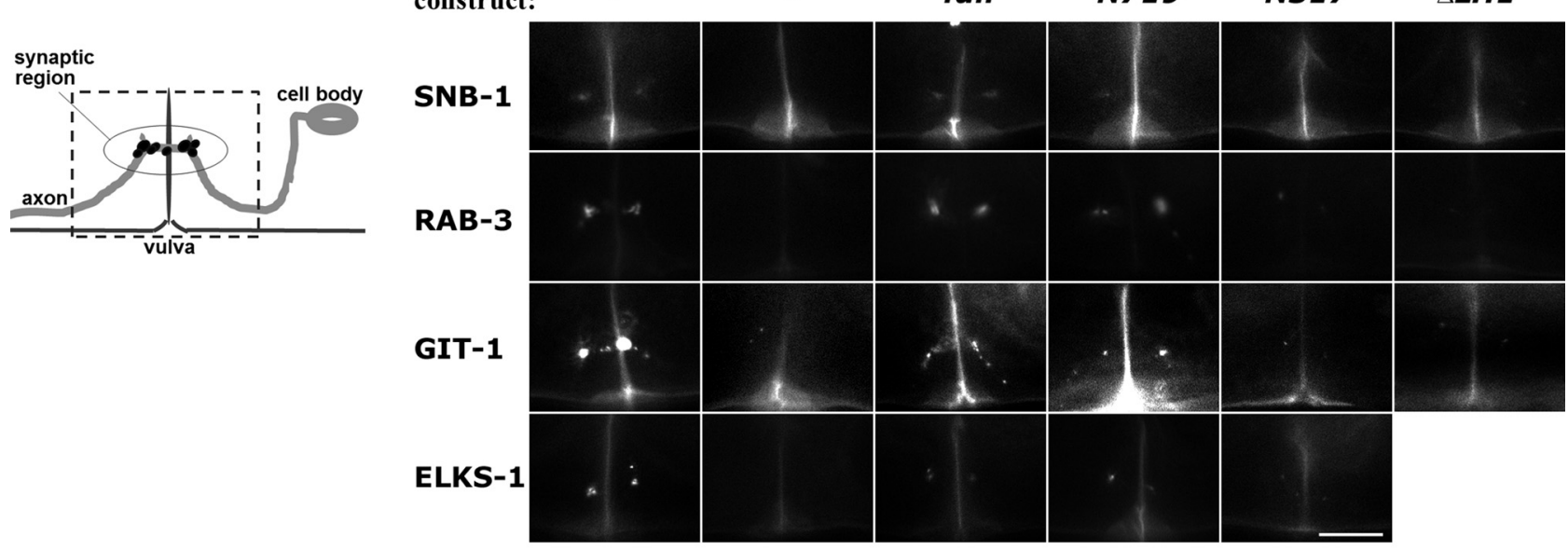

C

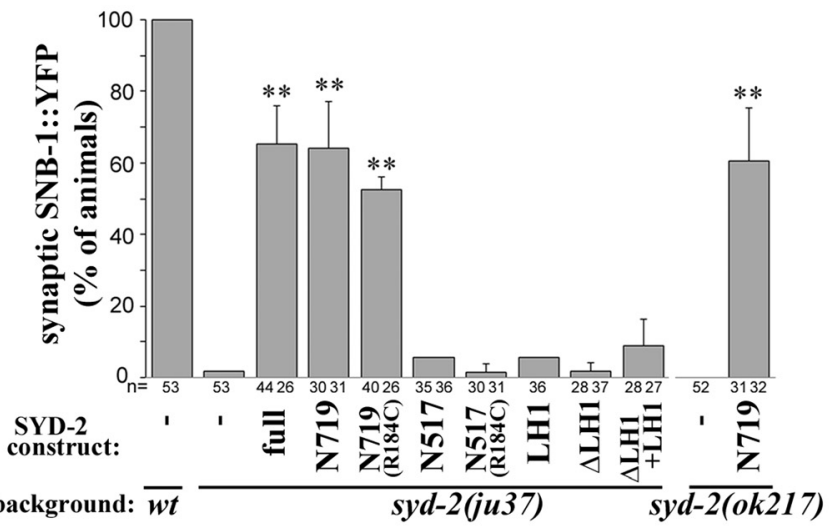

D
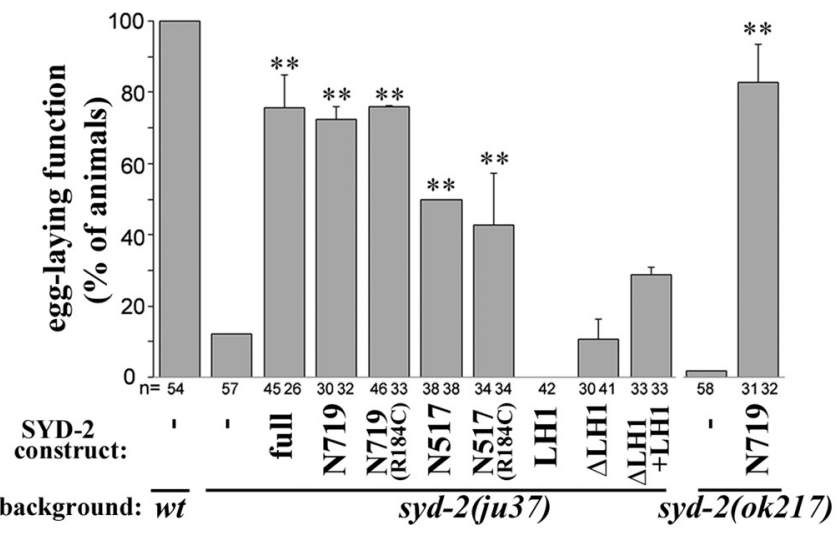

G

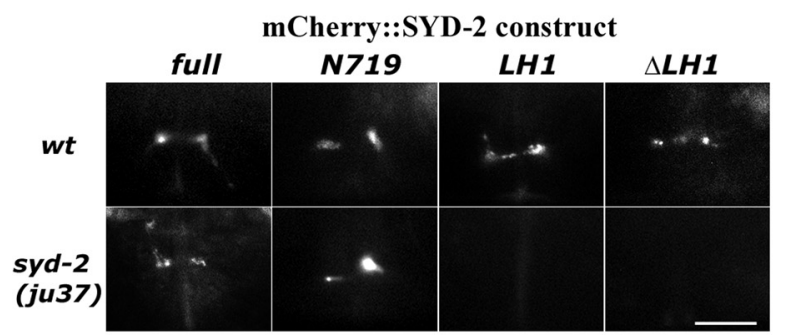

H

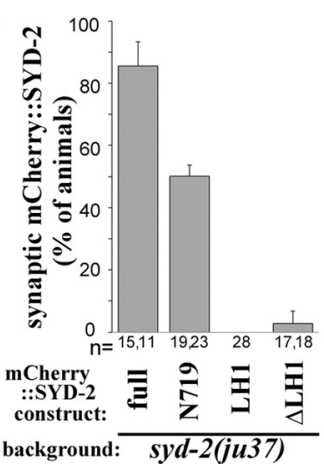

E

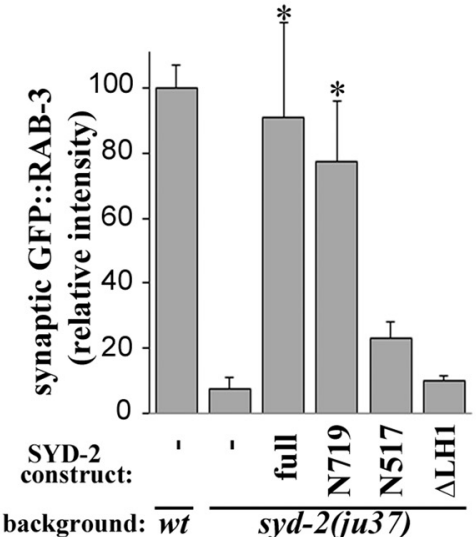

F
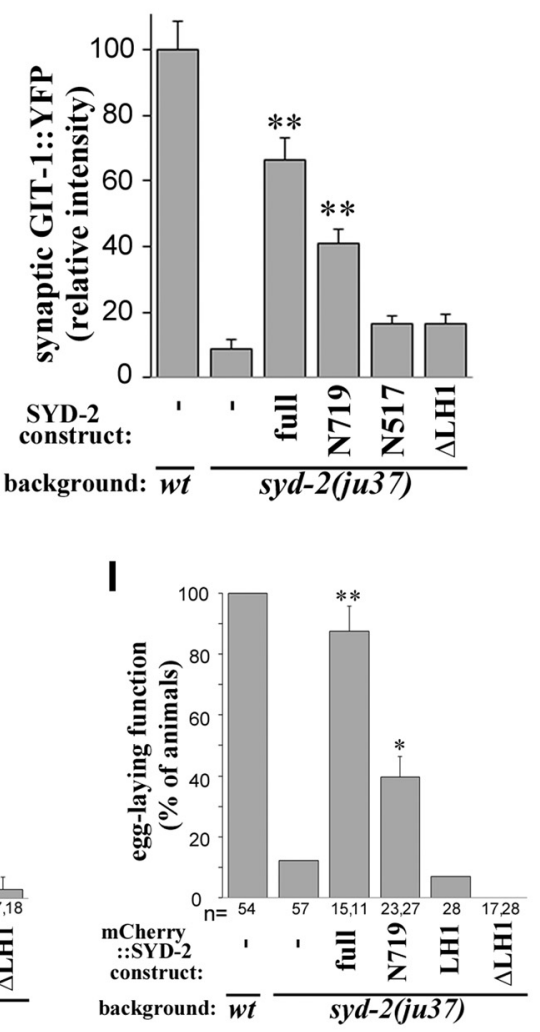

Figure 2. The conserved coiled-coil LH1 domain is necessary for SYD-2 function. $A$, Illustration of the HSN neuron and synapses. The neurite (bold gray line) normally forms synapses at vulva, which can be visualized as clusters of fluorescent synaptic markers (black dots). The dotted rectangle corresponds to the area shown in the images. $\boldsymbol{B}-\boldsymbol{F}$, Activity (Figure legend continues.) 
(Patel et al., 2006), juEx1206[P $\left.P_{\text {unc-86 }}-E L K S-1:: g f p ; P_{t t x-3}-r f p\right]$ (Dai et al.,

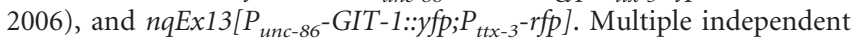
transgenic lines were obtained by microinjection following standard procedures (Mello et al., 1991), and one or two representative lines were crossed to different mutants or marker strains for analyses. Egg-laying function was assessed by scoring the number of $1 \mathrm{~d}$ adult hermaphrodites retaining older eggs after comma stage. Synaptic fluorescence markers were analyzed in live animals using Zeiss Axioplan2 microscopes and Nikon DS-QilMc. The peak fluorescent intensity (averaged for 10 pixels) of the brightest punctum at vulval synaptic region was measured in representative images using NIS-Elements software (Nikon).

Biochemistry. Recombinant GST-, His6-, and MBP-fusion proteins were produced in BL21(DE3) following incubation for $16 \mathrm{~h}$ at $20-22^{\circ} \mathrm{C}$. His6 and GST proteins were extracted by sonication in PBS, pH 7.4, with $0.1 \%$ Triton X-100, 1 mm DTT, and protease inhibitors (Roche), and purified with HisPur Cobalt resin (Thermo) and Glutathione-Sepharose (GE Healthcare), respectively, following the manufacturer's protocols with the buffer containing $0.1 \%$ Triton X-100 to avoid aggregation. For pull-down assay, supernatants containing $2.5 \mu \mathrm{g}$ of His6-tagged and 1, 5, or $25 \mu \mathrm{g}$ of GST-fusion proteins were incubated with HisPur Cobalt resin for $3 \mathrm{~h}$ at $4^{\circ} \mathrm{C}$. For chemical crosslinking, His6-SYD-2 proteins $(0.2 \mu \mathrm{g} /$ $\mu \mathrm{l})$ were incubated with glutaraldehyde $(0.0025$ or $0.005 \%)$ in PBS, pH 7.4, $150 \mathrm{~mm}$ imidazole, $0.1 \%$ Triton X-100, and $1 \mathrm{~mm}$ DTT for $5 \mathrm{~min}$ at $20^{\circ} \mathrm{C}$. Proteins were separated by SDS-PAGE, stained with Flamingo fluorescent gel stain (Bio-Rad), and detected with ImageQuant LAS4000 (GE Healthcare). For gel-filtration analysis, MBP-LH1 purified with Amylose resin (NEB) $(200 \mu \mathrm{g})$ was applied to an Agilent 1100 HPLC with PROTEIN KW-803 column (Shodex) equilibrated with $20 \mathrm{~mm}$ potassium phosphate, $\mathrm{pH} 6.8,50 \mathrm{~mm}$ sodium chloride at $0.5 \mathrm{ml} / \mathrm{min}$ flow rate, detected by DAWN HELEOS $8+$ and Optilab rEX (Wyatt).

COS-7 and CAD (Qi et al., 1997) cells were transfected with FLAGand EGFP-pcDNA3.1 plasmids in LipofectAMINE2000 (Invitrogen), and cultured for $24 \mathrm{~h}$. For Blue Native-PAGE analysis, COS-7 cells were lysed in PBS, pH 7.4, plus $0.1 \%$ Triton X-100 with protease inhibitors (Roche), and the supernatants were separated in NativePAGE 4-16\% Gel (Invitrogen), and detected by Western blotting with anti-FLAG M2 (Sigma-Aldrich). CAD cells were differentiated by serum deprivation, and observed with BZ-9000 microscope (Keyence) after $48 \mathrm{~h}$. We quantified the area of clusters with high levels of GFP signals in neurites by Image (NIH). All biochemical experiments were performed two or three times and reproducible.

\section{Results}

\section{LH1 domain is necessary for SYD-2 function in presynaptic assembly}

SYD-2/Liprin- $\alpha$ proteins share evolutionally conserved domain composition (Fig. 1A,B). To dissect the functional domains of SYD-2 by transgenic analysis, we focused on the $C$. elegans HSN neurons, which form en passant synapses onto VC neurons and vulval muscles at vulva region and control egg-laying behavior (Fig. 2A) (White et al., 1986; Shen and Bargmann, 2003). These

\footnotetext{
(Figure legend continued.) of pan-neurally expressed SYD-2 transgenic constructs to rescue syd-2(If) phenotype. $\boldsymbol{B}$, Localization of synaptic vesicle markers SNB-1::YFP and GFP::RAB-3, presynaptic active zone markers GIT-1::YFP and ELKS-1::GFP at vulval synaptic region in HSN in wild-type, syd-2(ju37) and syd-2(ju37) with SYD-2 transgenes. C, Quantification of SNB-1::YFP localization at HSN synapses. D, Quantification of egg-laying behavior. Values represent percentage of animals (mean $\pm S D$ of independent lines, and $n=$ the numbers of animals scored). $\boldsymbol{E}, \boldsymbol{F}$, Quantification of fluorescence intensity of GFP::RAB-3 $(\boldsymbol{E})$ and GIT-1::YFP $(\boldsymbol{F})$ puncta at synaptic regions. Values represent relative intensity (mean \pm SEM, 5 animals). G-I, Expression of mCherry-tagged SYD-2 transgenic constructs in HSN. G, Localization of mCherry::SYD-2 constructs (full-length, N719, LH1, and $\Delta \mathrm{LH} 1$ ) at HSN synaptic region in wild type and syd-2(ju37). H, Quantification of mCherry::SYD-2 localization. I, Quantification of egg-laying behavior. Values represent percentage of animals (mean $\pm S D$ of independent lines, $n=$ the numbers of animals scored). ${ }^{* *} p<0.01,{ }^{*} p<0.05$ [Fisher's exact test with Holm correction in $\mathbf{C}, \mathbf{D}, \mathbf{I}$, Dunnett's test in $\boldsymbol{E}, \boldsymbol{F}$ against syd-2(If)]. Scale bars, $10 \mu \mathrm{m}$.
}

synapses are visualized with fluorescence-tagged presynaptic protein reporters, such as synaptic vesicle proteins SNB-1 and RAB-3, and active zone components GIT- 1 and ELKS-1. In wildtype animals, fluorescent clusters for these reporters are distinctly visible at the vulva (Fig. $2 B$ ). In syd-2 loss-of-function animals, syd-2(ju37) and syd-2(ok217), the synaptic localizations of these markers are undetectable or largely diminished, and older-aged eggs are retained in their bodies as previously reported (Fig. 2B-F) (Dai et al., 2006; Patel et al., 2006).

We pan-neurally expressed a series of SYD-2 cDNA constructs to determine the region that is sufficient to rescue syd-2(lf) defects in HSN (Figs. 1C, 2B-F). Transgenic expression of fulllength SYD-2 rescued both synaptic markers and egg-laying defects. N-terminal 719 aa (N719), which contains the coiled-coil regions but lacks SAM domains, showed rescuing activity comparable to that of the full-length SYD-2 in both syd-2(ju37) and syd-2(ok217). Shorter N-terminal fragments, N517 and LH1, were insufficient for complete rescue. Expression of equivalent constructs containing the gain-of-function mutation R184C did not improve the degree of rescuing. However, removing LH1 domain from full-length SYD-2, SYD-2 $\Delta$ LH1, completely abolished rescuing activity. Coexpression of SYD-2 $\Delta \mathrm{LH} 1$ and LH1 showed no significant rescuing activity. To examine synaptic localization, we next expressed mCherry-tagged constructs in HSN. In syd-2(ju37), mCherry-tagged full-length and N719, but not SYD-2 $\Delta$ LH1 or LH1, rescued the egg-laying defects and displayed clusters of fluorescence at the synaptic region (Fig. $2 G-I$ ). In wild-type animals, all the mCherry-tagged constructs were localized properly to the synaptic region (Fig. $2 G$ ), possibly through interactions with endogenous SYD-2 or other presynaptic components. These results indicate that the $\mathrm{N}$-terminal coiled-coil region of SYD-2 has a core role for presynaptic assembly and the LH1 domain is essential for SYD-2 function.

\section{LH1 domain of SYD-2 is sufficient to restore the synaptic assembly in syd-1 mutant}

To further investigate how the domains contribute to the activities of SYD-2, we performed similar transgenic analyses in syd-1(lf) background (Figs. 1C, 3). syd-1(ju2) animals showed egglaying defects, and the clustering of synaptic markers at HSN synapses were undetectable or largely diminished as in syd-2(lf). Overexpression of full-length SYD-2 suppressed both defects, as reported previously (Dai et al., 2006; Patel et al., 2006). Expression of SYD-2 N terminus (N719 and N517) recapitulated this rescuing activity (Fig. $3 B, C$ ). Strikingly, expression of the LH1 domain alone also showed a comparable degree of rescuing activity (Fig. 3B-E). Introducing R184C to N719, N517, or LH1 did not enhance the degree of suppression. The LH1 domain of human Liprin- $\alpha 1$ (hLH1) is $82 \%$ identical to SYD-2LH1 (Fig. $1 A, B)$, and when expressed in C. elegans, also suppressed syd1(ju2) (Fig. 3B,C). Shorter fragments of LH1 displayed little or no suppression effects. Conversely, SYD-2 lacking the entire LH1 (SYD-2 $\Delta$ LH1) or 20 aa in LH1 $(\Delta 179-198)$ failed to suppress syd-1(ju2). As for their localization, mCherry-tagged LH1 was detectable at vulval synaptic site in syd-1(ju2), but SYD-2 LLH1 was not (Fig. $3 F)$. Taken together, these results indicate that the SYD-2 LH1 domain is necessary and sufficient to be localized at HSN synaptic sites and restore the synaptic assembly defects in syd-1(lf). These analyses also establish that in the syd-1 mutants the LH1 domain can act as an inducer to trigger wild-type SYD-2 function. 
A
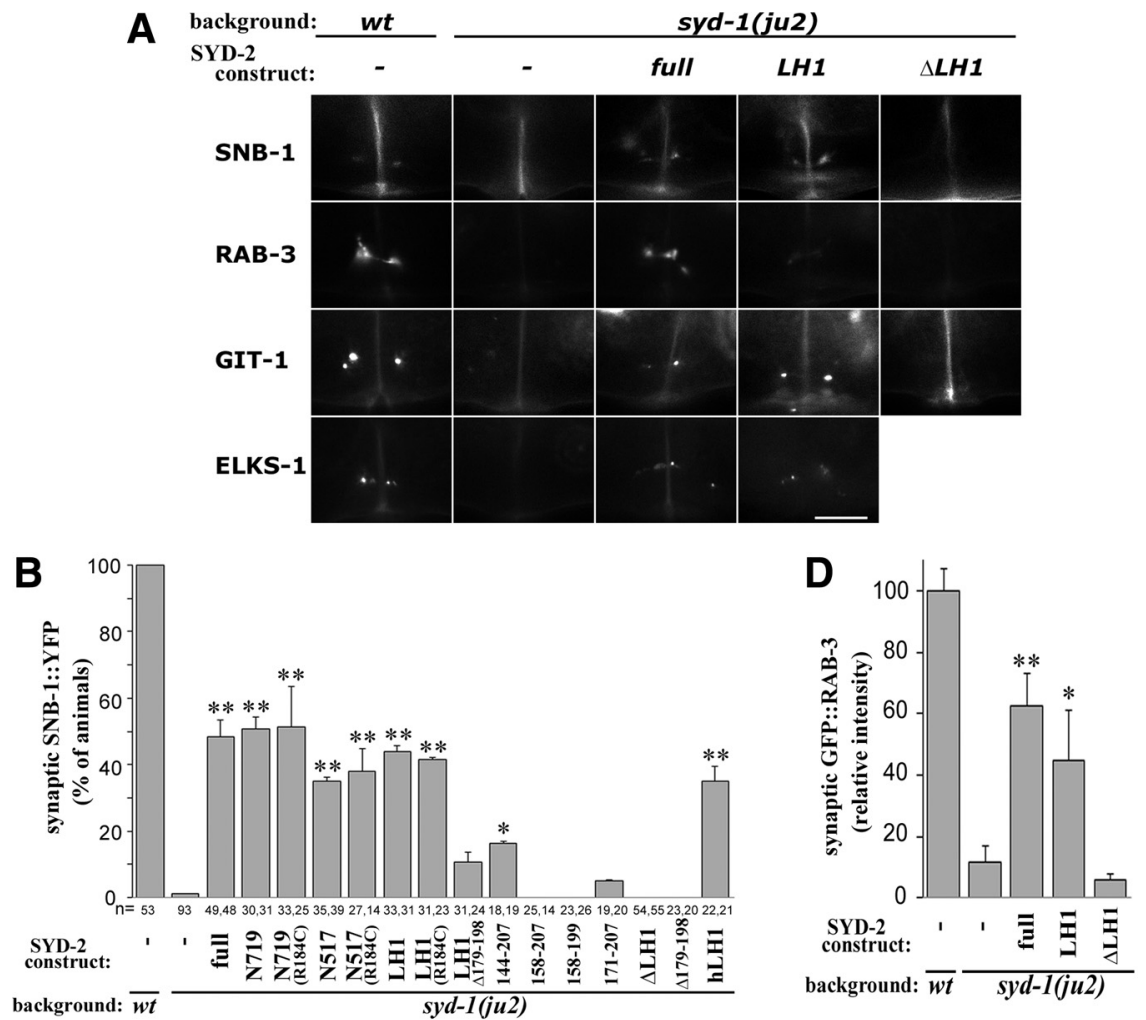

C
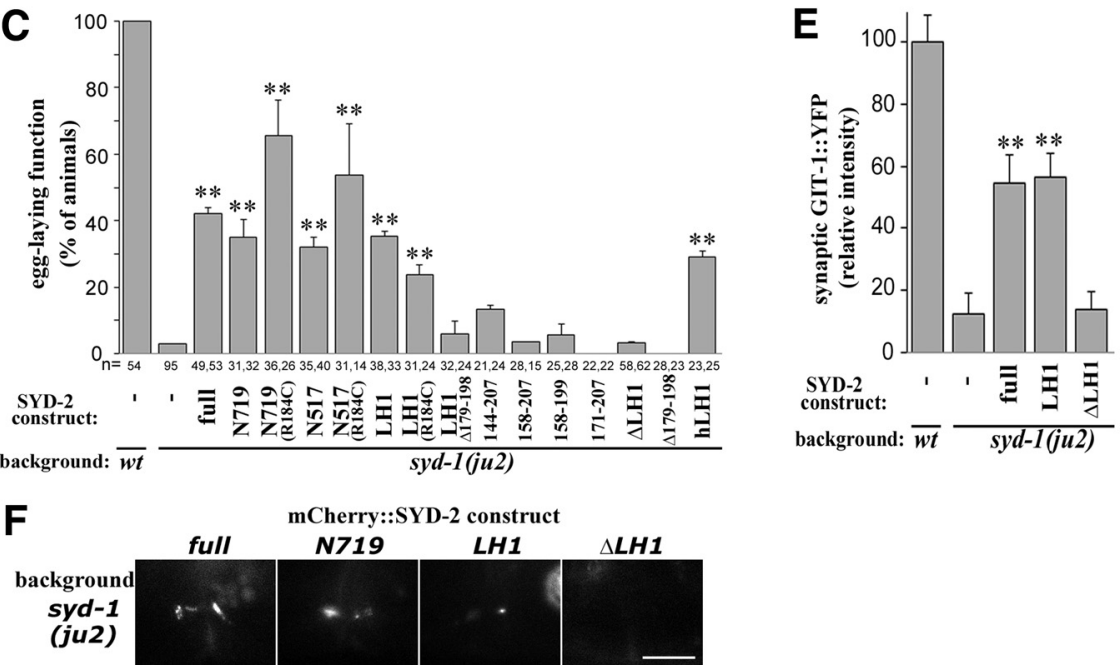

Figure 3. The LH1 domain of SYD-2 is necessary and sufficient for suppressing syd-1(If) phenotypes. A-E, Activity of panneurally expressed SYD-2 transgenic constructs to suppress syd-1(If) phenotype. $\boldsymbol{A}$, Localization of synaptic vesicle markers SNB-1::YFP and GFP::RAB-3, presynaptic active zone markers GIT-1::YFP and ELKS-1::GFP at vulval region in wild type, syd-1(juz), and syd-1(ju2) with SYD-2 transgenes. B, Quantification of SNB-1::YFP localization at HSN synapses. C, Quantification of egg-laying behavior. Values represent percentage of animals (mean $\pm S D$ of two independent lines, $n=$ the numbers of animals scored). $\boldsymbol{D}$, $\boldsymbol{E}$, Quantification of fluorescence intensity of GFP::RAB-3 (D) and GIT-1::YFP (E) puncta at synaptic region. Values represent relative intensity (mean \pm SEM, 5 animals). $\boldsymbol{F}$, Localization of mCherry::SYD-2 constructs (full-length, N719, LH1, and $\Delta$ LH1) at HSN synaptic region in syd-1(ju2). ${ }^{* *} p<0.01,{ }^{*} p<0.05$ [Fisher's exact test with Holm correction in $\boldsymbol{B}, \boldsymbol{C}$, Dunnett's test in $\boldsymbol{D}, \boldsymbol{E}$ against syd-1(ju2)]. Scale bars, $10 \mu \mathrm{m}$.

\section{Dimerization of LH1 domain of SYD-2/Liprin- $\alpha$ mediates homo-oligomerization}

The LH1 domain of SYD-2/Liprin- $\alpha$ is not known to be directly involved in binding to previously identified binding proteins of Liprin- $\alpha$, while it contains a motif recognized by the APC E3 ubiquitin ligase in Drosophila (van Roessel et al., 2004). As $\mathrm{N}$-terminal half of SYD-2/Liprin- $\alpha$ was reported to bind homophilically (Serra-Pagès et al., 1998; Wagner et al., 2009; Astigar- raga et al., 2010), we hypothesized that LH1 domain could mediate this homomeric interaction. To test this, we examined in vitro protein interactions by pulldown analysis. Recombinant His6-tagged SYD-2LH1 and GST-tagged SYD-2LH1 showed homomeric binding (Fig. 4A). SYD-2LH1 $\Delta 179-198$, which failed to suppress syd-1(lf) (Fig. $3 B, C$ ), significantly reduced the binding to $<10 \%$. Likewise, $\mathrm{LH} 1$ domain of human Liprin- $\alpha 1$ (hLH1) interacted with both GST-fused hLH1 and SYD2LH1 (Fig. 4B). The LH1 domains of all four human Liprin- $\alpha$, including Liprin- $\alpha 2$ and 3 , which are predominantly expressed in brain, share a high degree of sequence identity (Fig. $1 B$ ) and may share homomeric affinity.

To address the property of the homomeric interaction of LH1 domain, we performed chemical crosslinking. After crosslinking, in addition to $17 \mathrm{kDa}$ monomer, His6-tagged SYD-2LH1 was observed at $\sim 35 \mathrm{kDa}$ corresponding to the size of dimer (Fig. 4C). Under the same condition, the negative control proteins SYD-2 1-90 and $516-719$ produced only nonspecific crosslinked products. To further examine the native state of the LH1 domain at high concentration, we analyzed LH1 protein fused to monomeric maltose binding protein by gel filtration with multi-angle laser light scattering (MALS). The absolute molecular weight of the major peak of MBPLH1 was measured as $116 \mathrm{kDa}$ (Fig. $4 D$, arrowhead), nearly twofold larger than MBP-LH1 monomer (55 kDa), indicating that the LH1 domain can form a homomeric dimer in vitro.

To explore whether the dimeric property of the LH1 domain is important for enhancing SYD-2 activity in vivo, we constructed a chimeric SYD-2 mutant protein (SYD-2-DI), in which the LH1 was replaced with an artificial dimerization domain, GCN-IL (Fig. $1 C)$. GCN-IL is a derivative of the leucine zipper-based GCN4-LI peptides that forms a stable dimer and has been widely used as a constitutive dimerization domain (Harbury et al., 1993). Panneural expression of SYD-2-DI displayed partial suppression of syd-1(ju2), but failed to rescue $s y d-2(j u 37)$ phenotypes (Fig. 4E,F). These results support a conclusion that the role of the LH1 domain in SYD-2 activation can be complemented by constitutive dimerization in part, although the LH1 may likely also act through interactions besides simple dimerization.

SYD-2/Liprin- $\alpha$ at the synaptic region is likely clustered and integrated in large protein complex in vivo (Fouquet et al., 2009). To address the oligomeric property of SYD-2/Liprin- $\alpha$ coiled-coil regions, we expressed the $\mathrm{N}$ terminus of human 
A

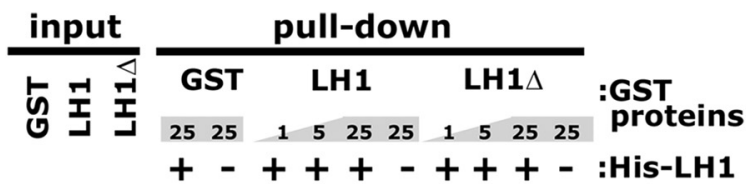

B

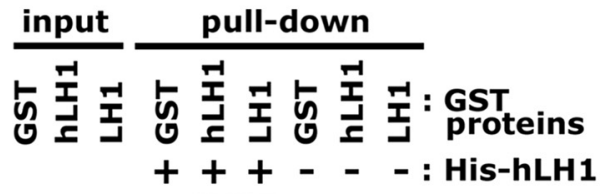

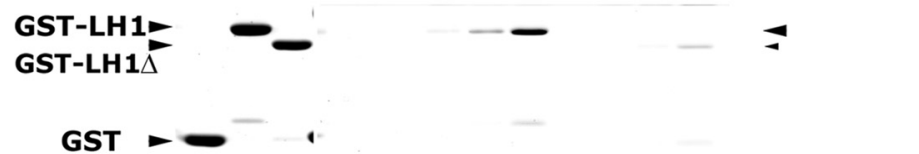

$\underset{\text { GST-hLH1 }}{\text { GST-LH1 }}$

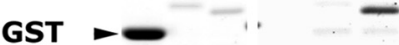

$\leftarrow$ His-LH1

$\leftarrow$ His-hLH1

C

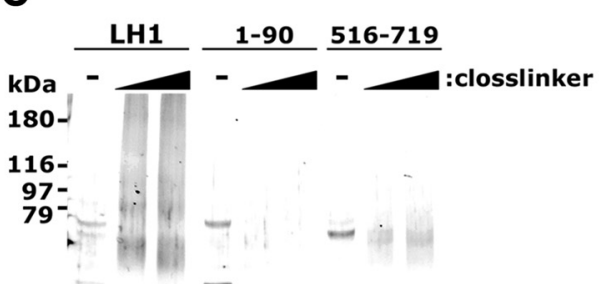

D

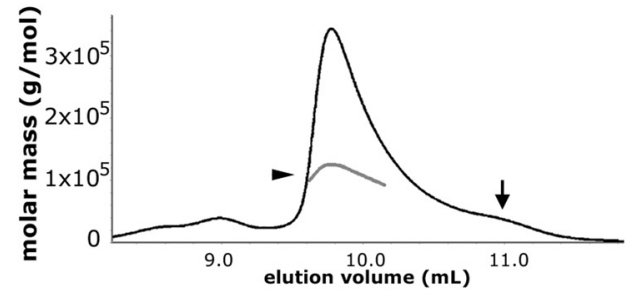

G

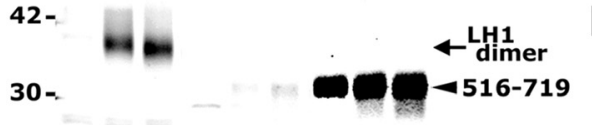

E

F 100
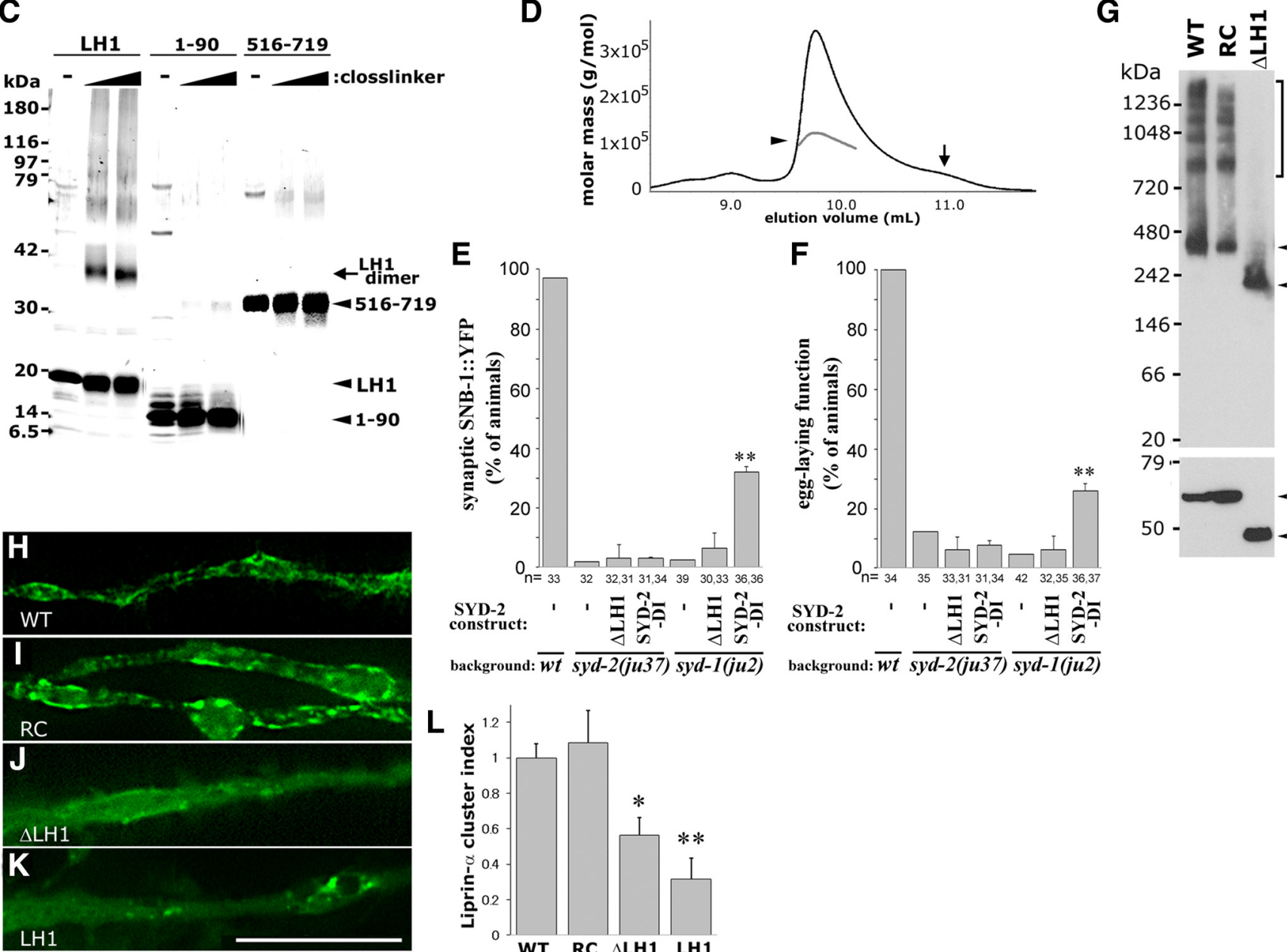

SYD-2
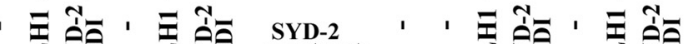

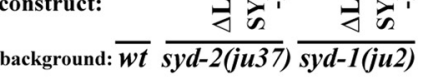

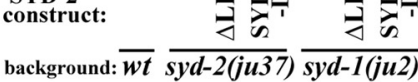

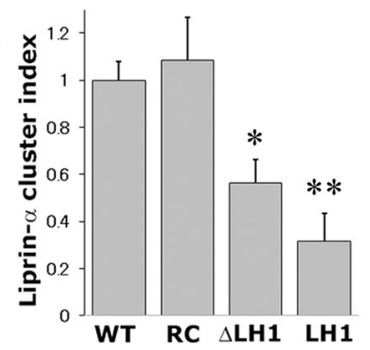

Figure 4. The LH1 domain of SYD-2/Liprin- $\alpha$ forms a dimer and is important for protein oligomerization and clustering. $A, B$, In vitro binding of LH1 domains of SYD-2 (LH1) and hLiprin- $\alpha 1$ (hLH1). GST-LH1, GST-LH1 $(\Delta 179-198)$, or GST was pulled down with His6-LH1 (A). GST-hLH1, GST-LH1, or GST was pulled down with His6-hLH1 (B). The numbers indicate the amount of input GST proteins (in micrograms). Eluate and input were analyzed by SDS-PAGE and staining. C, Chemical crosslinking of SYD-2LH1. His6-SYD-2(LH1), SYD-2(1-90), and SYD-2(516-719) were treated with glutaraldehyde, and analyzed by SDS-PAGE and staining. D, Measurement of absolute molecular mass of MBP-LH1 fusion protein. Chromatogram [refractive index (a.u.), black line] is overlaid with calculated molecular mass of the major dimeric peak by MALS (gray line, arrowhead). Arrow indicates peak position corresponding to monomer. $\boldsymbol{E}, \boldsymbol{F}$, Pan-neural expression of SYD-2-DI in syd-2(ju37) or syd-1(ju2). Quantification of SNB-1::YFP localization ( $\boldsymbol{E}$ ) and egg-laying behaviors $(\boldsymbol{F})$. Values represent percentage of animals (mean \pm SD of two independent lines, $n=$ the numbers of animals scored). ${ }^{* *} p<0.01$ (Fisher's exact test with Holm correction). G, BN-PAGE analysis of hLiprin- $\alpha 1$. FLAG-tagged hLiprin- $\alpha 1 \mathrm{~N}$-terminal proteins [aa 1-520; wild type, R181C, and $\Delta$ LH1 $(\Delta 88-214)]$ were size-separated in native condition by BN-PAGE (top) or in denatured condition by SDS-PAGE (bottom), and analyzed by Western blotting. $H-L$, Requirement of LH1 domain of hLiprin- $\alpha 1$ on the cluster formation in neuronal cells. GFP-tagged hLiprin- $\alpha 1$ full-length protein (H, wild type, $\boldsymbol{I}, \mathrm{R} 181 \mathrm{C}, \boldsymbol{J}, \Delta$ LH1) or LH1 domain $(\boldsymbol{K}, \mathrm{LH} 1)$ was expressed in CAD cells. Representative images of GFP fluorescence in neurites are shown. Scale bar, $20 \mu \mathrm{m}$. L, Quantification of total area of Liprin- $\alpha$ clusters in neurites. Values represent Liprin- $\alpha$ cluster index (mean \pm SEM of $5-6$ individual cells). ${ }^{* *} p<0.01,{ }^{*} p<0.05$ (Dunnett's test against wild type).

Liprin- $\alpha 1$ (Liprin- $\alpha 1 \mathrm{~N} ; 520$ aa) in COS-7 cells, since the heterologous expression of SYD-2 was very low (our unpublished data). We analyzed the oligomerization by Blue Native (BN)PAGE, a method allowing separation of protein complexes based on their size (Schägger and von Jagow, 1991). In BNPAGE, hLiprin- $\alpha 1 \mathrm{~N}$ (63 kDa in monomer) migrated as a discrete ladder with a main band at $\sim 380 \mathrm{kDa}$ (Fig. $4 G$ ), which is close to the size of a hexamer, although the molecular shape 
might obscure exact size estimation. The presence of discrete bands at even higher molecular weight further suggests that the $\mathrm{N}$ terminus of hLiprin- $\alpha 1$ is able to form large complexes of different multimer states with or without the help of endogenous binding partners. hLiprin- $\alpha 1 \mathrm{~N}$ R181C, corresponding to SYD-2 R184C, displayed a similar pattern (Fig. $4 G$ ). hLiprin- $\alpha 1 \mathrm{~N}$ lacking LH1 (hLiprin- $\alpha 1 \mathrm{~N} \Delta \mathrm{LH} 1: 48 \mathrm{kDa} /$ monomer) was predominantly detected at $\sim 200 \mathrm{kDa}$. Strikingly, hLiprin$\alpha 1 \mathrm{~N} \Delta \mathrm{LH} 1$ did not display discrete ladder-like bands at higher molecular weights (Fig. 4G). These results suggest that the LH1 domain is likely the core for the formation of higher oligomeric complexes, although multiple regions may be capable of mediating homomeric interactions of Liprin- $\alpha$.

To further examine the role of LH1 in the clustering of Liprin- $\alpha$ proteins, we analyzed the localization of GFP-tagged hLiprin- $\alpha 1$ in a mouse CNS cell line, CAD (Qi et al., 1997). Wild-type hLiprin- $\alpha 1$ and hLiprin- $\alpha 1$ (R181C) appeared as small fluorescent clusters in neurites especially close to the edge of cells (Fig. $4 H, I, L$ ), possibly associated with membrane structure as previously observed in non-neuronal cells (Serra-Pagès et al., 1998). In contrast, hLiprin- $\alpha 1$ lacking LH1 ( $\Delta$ LH1) showed more diffused cytoplasmic localization and had reduced numbers of clusters along neurites (Fig. 4J,L). LH1 alone was also mostly diffuse in neurites (Fig. $4 K, L$ ). Taken together, these results indicate that the LH1 domain in Liprin- $\alpha$ has a critical role in regulating the clustered localization of the proteins, probably via homomeric interaction.

\section{Discussion}

Among the presynaptic active zone proteins, SYD-2/Liprin- $\alpha$ proteins hold a central interest for their roles in promoting the assembly of presynaptic density (Spangler and Hoogenraad, 2007; Jin and Garner, 2008). Based on our structure-function and biochemical studies of SYD-2/Liprin- $\alpha$, we propose that self-association of SYD-2/Liprin- $\alpha$ through the short conserved coiled-coil segments is one of the key steps for presynaptic assembly.

Recent studies from C. elegans and Drosophila have shown that proper localization of SYD-2/DLiprin- $\alpha$ in nascent presynaptic sites depends on the function of SYD-1/DSyd-1. ELKS-1/ Bruchpilot binds both SYD-1/DSyd- 1 and SYD-2/DLiprin- $\alpha$, to mediate interactions with proteins functioning in synaptic vesicle release (Dai et al., 2006; Patel et al., 2006; Fouquet et al., 2009; Patel and Shen, 2009; Owald et al., 2010). In C. elegans HSN synapses, the requirement of SYD- 1 can be bypassed by either overexpression or the gain-of-function mutation of SYD-2, in part via enhancing the interaction of SYD-2 with ELKS-1 (Dai et al., 2006; Patel et al., 2006). These findings suggest that the amount of SYD-2 recruited to nascent presynaptic site and the property of SYD-2 to form a complex with its binding partners such as ELKS are important to trigger synaptic assembly and to promote synapse maturation. The $\mathrm{N}$-terminal coiled-coil region of SYD-2 binds multiple active zone proteins such as ELKS-1/ ELKS and UNC-10/RIM, and is the functional core for presynaptic assembly in HSN. Our biochemical analyses suggest that the dimerization of LH1 domain acts as a structural core and may likely organize the rest of the N-terminal coiled-coil segments to interact with each other, facilitating formation of higher-order oligomers as well as interacting with other binding proteins. Overexpression of LH1 domain may enable endogenous SYD-2 to form stable functional complex, thereby overcoming the deficiency of SYD-1. The incomplete rescue of syd-2(lf) by the constitutive dimer also indicates that LH1 domain has additional roles besides simple dimerization; for example, LH1 may interact with particular proteins or lipids. Alternatively, unique structure or association/dissociation balance of the LH1 dimer may be critical for full function of SYD-2.

Although no structural analysis of the coiled-coil-rich regions of SYD-2/Liprin- $\alpha$ has been reported, parallel dimer structure of the short coiled-coil region of Liprin- $\beta$ was recently reported (Stafford et al., 2011). Future experiments to elucidate the conformation and structure of SYD-2/Liprin- $\alpha$, as well as exact oligomerization state in vivo, will be important to better understand this molecular machinery.

\section{References}

Astigarraga S, Hofmeyer K, Farajian R, Treisman JE (2010) Three Drosophila liprins interact to control synapse formation. J Neurosci 30:15358-15368.

Brenner S (1974) The genetics of Caenorhabditis elegans. Genetics 77: 71-94.

Dai Y, Taru H, Deken SL, Grill B, Ackley B, Nonet ML, Jin Y (2006) SYD-2 Liprin-alpha organizes presynaptic active zone formation through ELKS. Nat Neurosci 9:1479-1487.

Fouquet W, Owald D, Wichmann C, Mertel S, Depner H, Dyba M, Hallermann S, Kittel RJ, Eimer S, Sigrist SJ (2009) Maturation of active zone assembly by Drosophila Bruchpilot. J Cell Biol 186:129-145.

Hallam SJ, Goncharov A, McEwen J, Baran R, Jin Y (2002) SYD-1, a presynaptic protein with PDZ, C2 and rhoGAP-like domains, specifies axon identity in C. elegans. Nat Neurosci 5:1137-1146.

Harbury PB, Zhang T, Kim PS, Alber T (1993) A switch between two-, three-, and four-stranded coiled coils in GCN4 leucine zipper mutants. Science 262:1401-1407.

Jin Y, Garner CC (2008) Molecular mechanisms of presynaptic differentiation. Annu Rev Cell Dev Biol 24:237-262.

Kaufmann N, DeProto J, Ranjan R, Wan H, Van Vactor D (2002) Drosophila liprin-alpha and the receptor phosphatase Dlar control synapse morphogenesis. Neuron 34:27-38.

Ko J, Na M, Kim S, Lee JR, Kim E (2003) Interaction of the ERC family of RIM-binding proteins with the liprin-alpha family of multidomain proteins. J Biol Chem 278:42377-42385.

Mello CC, Kramer JM, Stinchcomb D, Ambros V (1991) Efficient gene transfer in C. elegans: extrachromosomal maintenance and integration of transforming sequences. EMBO J 10:3959-3970.

Owald D, Fouquet W, Schmidt M, Wichmann C, Mertel S, Depner H, Christiansen F, Zube C, Quentin C, Körner J, Urlaub H, Mechtler K, Sigrist SJ (2010) A Syd-1 homologue regulates pre- and postsynaptic maturation in Drosophila. J Cell Biol 188:565-579.

Patel MR, Shen K (2009) RSY-1 is a local inhibitor of presynaptic assembly in C. elegans. Science 323:1500-1503.

Patel MR, Lehrman EK, Poon VY, Crump JG, Zhen M, Bargmann CI, Shen K (2006) Hierarchical assembly of presynaptic components in defined $C$. elegans synapses. Nat Neurosci 9:1488-1498.

Qi Y, Wang JK, McMillian M, Chikaraishi DM (1997) Characterization of a CNS cell line, CAD, in which morphological differentiation is initiated by serum deprivation. J Neurosci 17:1217-1225.

Schägger H, von Jagow G (1991) Blue native electrophoresis for isolation of membrane protein complexes in enzymatically active form. Anal Biochem 199:223-231.

Schoch S, Castillo PE, Jo T, Mukherjee K, Geppert M, Wang Y, Schmitz F, Malenka RC, Südhof TC (2002) RIMlalpha forms a protein scaffold for regulating neurotransmitter release at the active zone. Nature 415:321-326.

Serra-Pagès C, Medley QG, Tang M, Hart A, Streuli M (1998) Liprins, a family of LAR transmembrane protein-tyrosine phosphatase-interacting proteins. J Biol Chem 273:15611-15620.

Shen K, Bargmann CI (2003) The immunoglobulin superfamily protein SYG-1 determines the location of specific synapses in C. elegans. Cell 112:619-630

Spangler SA, Hoogenraad CC (2007) Liprin-alpha proteins: scaffold molecules for synapse maturation. Biochem Soc Trans 35:1278-1282.

Stafford RL, Tang MY, Sawaya MR, Phillips ML, Bowie JU (2011) Crystal 
structure of the central coiled-coil domain from human liprin-beta2. Biochemistry 50:3807-3815.

Stigloher C, Zhan H, Zhen M, Richmond J, Bessereau JL (2011) The presynaptic dense projection of the Caenorhabditis elegans cholinergic neuromuscular junction localizes synaptic vesicles at the active zone through SYD-2/liprin and UNC-10/RIM-dependent interactions. J Neurosci 31:4388-4396.

van Roessel P, Elliott DA, Robinson IM, Prokop A, Brand AH (2004) Independent regulation of synaptic size and activity by the anaphasepromoting complex. Cell 119:707-718.

Wagner OI, Esposito A, Köhler B, Chen CW, Shen CP, Wu GH, Butkevich E, Mandalapu S, Wenzel D, Wouters FS, Klopfenstein DR (2009) Synaptic scaffolding protein SYD-2 clusters and activates kinesin-3 UNC-104 in C. elegans. Proc Natl Acad Sci U S A 106:19605-19610.

White JG, Southgate E, Thomson JN, Brenner S (1986) The structure of the nervous system of the nematode Caenorhabditis elegans. Philos Trans R Soc Lond B Biol Sci 314:1-340.

Yeh E, Kawano T, Weimer RM, Bessereau JL, Zhen M (2005) Identification of genes involved in synaptogenesis using a fluorescent active zone marker in Caenorhabditis elegans. J Neurosci 25:3833-3841.

Zhai RG, Bellen HJ (2004) The architecture of the active zone in the presynaptic nerve terminal. Physiology (Bethesda) 19:262-270.

Zhen M, Jin Y (1999) The liprin protein SYD-2 regulates the differentiation of presynaptic termini in C. elegans. Nature 401:371-375. 